\title{
Pre-operative clinical predictors for cardiology referral prior to total joint arthroplasty: the 'asymptomatic' patient
}

Yassin Elsiwy ${ }^{1,2}$, Tristan Symonds ${ }^{2}$, Kenji Doma ${ }^{2,3}$, Kaushik Hazratwala ${ }^{1,2}$, Matthew Wilkinson ${ }^{2,4}$ and Hayley Letson ${ }^{1 *}$ (D)

\begin{abstract}
Background: No validated pre-operative cardiac risk stratification tool exists that is specific for total hip and total knee arthroplasty (THA and TKA, respectively). To reduce the risk of post-operative cardiac complication, surgeons need clear guidance on which patients are likely to benefit from pre-operative cardiac optimisation. This is particularly important for asymptomatic patients, where the need is harder to determine.

Methods: Primary THA and TKA performed between January 1, 2010, and December 31, 2017, were identified from a single orthopaedic practice. Over 25 risk factors were evaluated as predictors for patients requiring additional cardiac investigation beyond an ECG and echocardiogram, and for cardiac abnormality detected upon additional investigation. A multivariate logistic regression was conducted using significant predictor variables identified from inferential statistics. A series of predictive scores were constructed and weighted to identify the influence of each variable on the ability to predict the detection of cardiac abnormality pre-operatively.

Results: Three hundred seventy-four patients were eligible for inclusion. Increasing age $(p<0.001)$, history of cerebrovascular accident $(p=0.018$ ), family history of cardiovascular disease (FHx of CVD) $(p<0.001)$ and decreased ejection fraction (EF) $(p<0.001)$ were significant predictors of additional cardiac investigation being required. Increasing age $(p=0.003)$, male gender $(p=0.042)$, FHx of CVD $(p=0.001)$ and a reduced EF $(p<0.001)$ were significantly predictive for the detection of cardiac abnormality upon additional cardiac investigation.

* Correspondence: Hayley.letson@jcu.edu.au

${ }^{1}$ College of Medicine and Dentistry, James Cook University, 1 James Cook Drive, Townsville, QLD 4811, Australia

Full list of author information is available at the end of the article

C The Author(s). 2020 Open Access This article is licensed under a Creative Commons Attribution 4.0 International License, which permits use, sharing, adaptation, distribution and reproduction in any medium or format, as long as you give appropriate credit to the original author(s) and the source, provide a link to the Creative Commons licence, and indicate if changes were made. The images or other third party material in this article are included in the article's Creative Commons licence, unless indicated otherwise in a credit line to the material. If material is not included in the article's Creative Commons licence and your intended use is not permitted by statutory regulation or exceeds the permitted use, you will need to obtain permission directly from the copyright holder. To view a copy of this licence, visit http://creativecommons.org/licenses/by/4.0/ The Creative Commons Public Domain Dedication waiver (http://creativecommons.org/publicdomain/zero/1.0/) applies to the data made available in this article, unless otherwise stated in a credit line to the data. 
(Continued from previous page)

Conclusions: Increasing age, male gender, FHx of CVD and decreased ejection fraction are important risk factors to consider for pre-operative cardiac optimisation in THA and TKA patients. These findings can be applied towards future predictive models, to determine which asymptomatic patients are likely to benefit from pre-operative cardiac referral.

Keywords: Cardiac, Arthroplasty, TKA, THA, Risk factor, Complication

\section{Background}

Total joint arthroplasty (TJA) is the mainstay of treatment for end-stage osteoarthritis (OA) of the hip and knee, improving pain, function and quality of life [1]. Cardiac complication represents a major cause of morbidity and mortality after TJA and is associated with increased hospital mortality, length of stay and health expenditure [2-6]. Though rates of major cardiac complication are reported as low as $0.2-0.8 \%$, this risk becomes increasingly significant given the projected 174 and $673 \%$ increase in total hip (THA) and total knee (TKA) arthroplasty, respectively, by 2030 in the USA [2, 7-13].

Previous studies have identified risk factors for postoperative cardiac complication in TKA and THA cohorts; however, a recent systematic literature review identified several limitations within the current literature [14]. Firstly, there is inconsistency regarding which risk factors are significant predictors of cardiac complication. Secondly, few studies extended their findings by way of a predictive model of multiple significant risk factors that can be adopted for cardiac risk stratification prior to TJA. Although Waterman and colleagues proposed one such model using age $\geq 80$ years, history of cardiac disease, and hypertension, this tool has not been validated and further work is required to determine the value of other important risk factors in pre-operative cardiac risk stratification [3]. Therefore, no validated, orthopaedic-specific model(s) currently exist to facilitate pre-operative cardiac risk stratification. Although pre-operative referral for cardiac optimisation is clear-cut for the symptomatic patient already exhibiting signs of cardiovascular disease [15], the indication is less clear for asymptomatic patients not yet exhibiting clinical manifestations of a potential underlying and established cardiac disease.

The purpose of this study was to determine which risk factors should prompt orthopaedic surgeons to refer 'asymptomatic' patients for cardiac optimisation prior to TKA or THA. The primary objective being to identify risk factors which can be indicative of underlying and undiagnosed cardiac disease in an asymptomatic patient, which may otherwise place the patient at risk of cardiac complication peri- or post-TJA. Based on prior analysis, we hypothesised that age and history of cardiac disease will be significant predictors. However, we also sought to further determine the predictive value of additional potentially important risk factors [14]. Significant risk factors identified in this study may contribute to the development of a more comprehensive cardiac risk stratification tool that may better guide pre-operative cardiac referral for the 'asymptomatic' TJA patient.

\section{Methods}

This study was approved by the Mater Health Services North Queensland Human Research Ethics Committee (Approval: MHS20180424-01). The study was a retrospective consecutive short series of data collected between January 1, 2010, and December 30, 2017, at a single orthopaedic practice in Australia. Patients eligible for inclusion were those who underwent a TKA or THA and received pre-operative cardiology assessment including an electrocardiogram (ECG) and echocardiogram (ECHO) as a baseline investigation. Pre-operative cardiology assessment was standard practice for all patients, regardless of suspected risk, for this institution. Surgery was performed by a single surgeon and pre-operative cardiology review was completed by one of seven cardiologists. Patients who underwent bilateral TKA or THA, revision arthroplasty, or had a surgical indication other than osteoarthritis were excluded. As this study aims to risk-stratify within an asymptomatic TJA population, patients identified to have symptomatic cardiac disease including angina, evidence of heart failure (New York Heart Association class >1), orthopnoea, paroxysmal nocturnal dyspnoea, and/or peripheral oedema, at the initial surgical consultation, were excluded. A flowchart of the inclusion and exclusion criteria is provided as Supplementary Figure 1.

Data was collected by two investigators from electronic records. Variables were selected based on a prior systematic review examining risk factors for cardiac complication after TKA and THA [14]. The predictor variables included patient demographics; medical history (cardiac and noncardiac); family history of cardiac disease (FHx of CVD), defined as history of myocardial infarction, cardiac arrest, sudden cardiac death, ischaemic heart disease, coronary artery disease or congestive heart failure in a first-degree relative; and results of baseline cardiac investigation (ECG and $\mathrm{ECHO}$ ). Data extracted were age, gender, body mass index (BMI), diabetes, hypertension (HTN), hypercholesterolaemia, smoking history, alcohol history, chronic kidney disease, chronic obstructive pulmonary disease, asthma, obstructive sleep apnoea, chronic heart failure, myocardial infarction, coronary artery disease (CAD), 
valvular disease, arrhythmia, peripheral vascular disease, cerebrovascular accident (CVA) and venous thromboembolism [14]. The outcome variables for this analysis were (1) additional cardiac investigation (ACI) ordered by cardiologist (e.g. angiogram, CT-coronary angiography (CTCA), stress test, or myocardial perfusion scan (MIBI)) and (2) abnormality detected upon additional cardiac investigation (ADACI). Post-operative cardiac complications (defined as cardiac event within 30 days), as well as surgical delays (defined as a change in the date of surgery for cardiac optimisation), and surgical cancellation by cardiologist, were also recorded.

\section{Statistical methodology}

Data analysis was performed using SPSS 24.0 (IBM) with the alpha level set at 0.05 . The measure of central tendency and dispersion was reported as mean \pm standard deviation, and prevalence as frequencies. Based on Shapiro-Wilk test, continuous parameters were logtransformed prior to inferential statistical analyses. The discriminant and predictive capacities for $\mathrm{ACI}$ and ADACI were assessed using a three-tiered approach (Supplementary Figure 2). Firstly, an independent $t$ test was used to determine differences in age, BMI and ejection fraction (EF) between patients with, and without, the need for ACI, and between patients with, and without, ADACI. Similar inter-individual comparisons were conducted for all other nominal variables using a chisquared test of independence. Crude odds ratio and associated $95 \%$ confidence interval (CI) of all continuous and nominal variables were calculated using MedCalc (Version 19.0.7, Belgium). Secondly, a stepwise, multivariate logistic regression was conducted to determine whether the variables that were incorporated in the inferential statistics (i.e. independent variables) predicted ACI and ADACI (i.e. dependent variables). Adjusted odds ratio and associated 95\% CI derived from the multivariate logistic regression were also reported. According to the Hosmer and Lemeshow test, the dependent variables were not significant $(p$ $>0.05)$, demonstrating appropriate goodness of fit for the multivariable logistic regression, and that the sample size provided sufficient predictive capacity for the model. Finally, the variance inflation factor of both multivariate logistic regression models were $<5$ for the independent variables, indicating that the predictors demonstrated minimal multi-collinearity [16].

Internal validation of significant predictor variables for ADACI Based on the performance of predictor variables in the multivariate logistic regression model, a series of predictive scores were constructed that weighted the influence of each predictor on the odds of a cardiac abnormality being detected upon additional cardiac investigation. Numerical variables were normalised against the mean of the cohort, while categorical variables were allocated an arbitrary value of 1 for presence of the risk factor and 0 for its absence. To determine the discriminant capacity for ADACI, receiver operator characteristics (ROC) curves were generated to compute the area under the curve or cstatistic (c-statistic; 95\% CI). The model weighted predictors based on the odds of an abnormality detected upon additional cardiac investigation to closely reflect each risk factors' weight in the predictive model. Risk factors with an odds ratio $1 \leq \mathrm{OR}<2$ were multiplied by 1 point, $2 \leq$ OR $<3$ multiplied by 2 points, and $\geq 3$ multiplied by 3 points to produce a final score. The discriminative capability of the model was evaluated using the c-statistic. The stepwise, multivariate logistic regression allowed selection of pertinent predictors for the ROC curves. Appropriate model fitting of these explanatory variables were assessed via Hosmer-Lemeshow goodness of fit test, in conjunction with the likelihood-ratio chi-squared test. Accordingly, the results of the tests were not statistically significant, indicating that the statistical model was well calibrated, and our model improved from the null model. Furthermore, the Akaike information criterion was examined across several iterations as part of the stepwise process, with the model exhibiting the smallest value selected to ensure a better-fit model. The assumption of linearity of the multivariate logistic regression between the $\log$ odds and predictor variables was assessed using the Box-Tidwell test in Stata (version 16, Texas). With regard to the ROC curves, the cut-off demonstrating the greatest sensitivity and specificity was chosen.

\section{Results}

\section{Cohort composition and patient characteristics}

A total of 374 patients were eligible for inclusion. Four hundred forty-three patients were directly identified based on the inclusion criteria, though THA and TKA are not the only procedures performed at this centre. From a total of 443 patients, 7 patients were excluded due to cancellation of surgery for non-medical reasons and 62 patients were excluded due to missing crucial data, namely ECG and ECHO reports, which were unable to be retrieved from medical records (Supplementary Figure 1). Of the 374 patients eligible for inclusion, 272 (72.7\%) underwent TKA, and 102 (27.3\%) underwent THA (Additional Table 1). The mean age of the cohort was $69.9 \pm 9.0$ years, and the majority of patients were male (54.0\%). Hypertension was the most common risk factor (65.2\%), followed by hypercholesterolaemia (57.8\%) and diabetes (15.0\%) (Additional Table 1). More than one third $(35.3 \%)$ of patients reported a previous cardiac history, and 19.3\% reported a FHx of CVD. There were 22 (5.9\%) current smokers and 91 (24.3\%) ex-smokers in this population. The mean EF was 63.82 $\pm 8.28 \%$, and an ECG abnormality was found in 115 
Table 1 Risk factors associated with additional cardiac investigation

\begin{tabular}{|c|c|c|c|c|c|c|c|c|}
\hline \multirow[t]{2}{*}{ Variable } & \multicolumn{2}{|l|}{$\mathrm{ACl}$} & \multicolumn{6}{|c|}{ Statistics } \\
\hline & $\begin{array}{l}\text { Yes } \\
(n=138)\end{array}$ & $\begin{array}{l}\text { No } \\
(n=236)\end{array}$ & $x^{2}$ & $p$ value & z score & Crude OR & $95 \% \mathrm{Cl}$ & $p$ value \\
\hline \multicolumn{9}{|l|}{ Demographics } \\
\hline Age (years) & $72.3 \pm 8.7$ & $68.5 \pm 8.9$ & - & $<0.001$ & - & 1.05 & $1.03-1.08$ & $<0.001$ \\
\hline Male & $81(58.7 \%)$ & $121(51.3 \%)$ & 1.93 & 0.164 & 1.4 & 1.35 & $0.88-2.06$ & 0.170 \\
\hline Female & $57(41.3 \%)$ & 115 (48.7\%) & 1.93 & 0.164 & -1.4 & 0.74 & $0.48-1.13$ & 0.170 \\
\hline $\mathrm{BMI}$ & $31.4 \pm 5.5$ & $31.1 \pm 5.3$ & - & 0.592 & - & 1.01 & $0.97-1.05$ & 0.590 \\
\hline \multicolumn{9}{|l|}{ Past medical Hx } \\
\hline Diabetes & $27(19.6 \%)$ & $29(12.3 \%)$ & 3.62 & 0.057 & 1.9 & 1.74 & $0.98-3.08$ & 0.060 \\
\hline Hypertension & 105 (76.1\%) & 139 (58.9\%) & 11.35 & 0.001 & 3.4 & 2.22 & $1.39-3.55$ & $<0.001$ \\
\hline Hypercholesterolaemia & $88(63.8 \%)$ & $128(54.2 \%)$ & 3.24 & 0.072 & 1.8 & 1.49 & $0.96-2.29$ & 0.070 \\
\hline Smoking & $11(8.0 \%)$ & $11(4.7 \%)$ & 5.97 & 0.050 & 1.3 & 2.03 & $0.85-4.88$ & 0.110 \\
\hline Ex-smoker & $41(29.7 \%)$ & $50(21.2 \%)$ & 5.97 & 0.050 & 1.9 & 1.67 & $1.03-2.7$ & 0.040 \\
\hline Alcohol & $13(9.4 \%)$ & $29(12.3 \%)$ & 0.72 & 0.397 & -0.8 & 0.74 & $0.37-1.48$ & 0.400 \\
\hline CKD & $12(8.7 \%)$ & 11 (4.7\%) & 2.47 & 0.292 & 1.6 & 1.95 & $0.83-4.54$ & 0.120 \\
\hline Asthma & $16(11.6 \%)$ & $28(11.9 \%)$ & 0.006 & 0.938 & -0.1 & 0.97 & $0.51-1.87$ & 0.940 \\
\hline COPD & $7(5.1 \%)$ & 7 (3.0\%) & 1.07 & 0.300 & 1.0 & 1.75 & $0.60-5.09$ & 1.000 \\
\hline OSA & $13(9.4 \%)$ & $3(12.7 \%)$ & 0.93 & 0.336 & -1.0 & 0.71 & $0.36-1.42$ & 0.340 \\
\hline \multicolumn{9}{|l|}{ Past CV Hx } \\
\hline Cardiac Hx total & $63(45.7 \%)$ & $69(29.2 \%)$ & 10.27 & 0.001 & 3.2 & 2.03 & $1.31-3.15$ & 0.002 \\
\hline CCF & $3(2.2 \%)$ & $5(2.1 \%)$ & 0.001 & 0.972 & 0.0 & 1.03 & $0.24-4.36$ & 0.970 \\
\hline $\mathrm{Ml}$ & $14(10.1 \%)$ & 14 (5.9\%) & 2.23 & 0.135 & 1.5 & 1.79 & $0.83-3.88$ & 0.140 \\
\hline$C A D$ & $38(27.5 \%)$ & $36(15.3 \%)$ & 8.28 & 0.004 & 2.9 & 2.11 & $1.26-3.53$ & 0.005 \\
\hline Valvular disease & $10(7.2 \%)$ & 15 (6.4\%) & 0.11 & 0.739 & 0.3 & 1.15 & $0.50-2.64$ & 0.740 \\
\hline Arrhythmia & $25(18.1 \%)$ & $30(12.7 \%)$ & 2.04 & 0.154 & 1.4 & 1.52 & $0.85-2.71$ & 0.160 \\
\hline PVD & $4(2.9 \%)$ & $3(1.3 \%)$ & 1.26 & 0.262 & 1.1 & 2.32 & $0.51-10.52$ & 0.280 \\
\hline CVA & 19 (13.8\%) & $8(3.4 \%)$ & 14.00 & $<0.001$ & 3.7 & 4.55 & $1.93-10.70$ & $<0.001$ \\
\hline VTE & $8(5.8 \%)$ & $6(2.5 \%)$ & 2.56 & 0.110 & 1.6 & 2.36 & $0.80-6.95$ & 0.120 \\
\hline Family Hx of cardiac disease & $42(30.4 \%)$ & $30(12.7 \%)$ & 17.60 & $<0.001$ & 4.2 & 3.00 & $1.77-5.09$ & $<0.0001$ \\
\hline \multicolumn{9}{|l|}{ Baseline cardiac Ix } \\
\hline ECG abnormality & $53(38.4 \%)$ & $62(26.3 \%)$ & 6.02 & 0.014 & 2.5 & 1.75 & $1.12-2.74$ & 0.001 \\
\hline Ejection fraction (\%) & $62.0 \pm 10.2$ & $64.9 \pm 6.7$ & - & 0.005 & - & 0.96 & $0.93-0.99$ & 0.002 \\
\hline
\end{tabular}

Numerical predictor variables (age, BMl, ejection fraction) are presented as mean \pm standard deviation and were assessed using an independent $t$ test. Categorical predictor variables are presented as frequencies and were assessed using chi-squared test of independence. The crude ratio and associated $95 \% \mathrm{Cl}$ are also reported. Significant predictor variables are indicated by $p<0.05$

$A C l$ additional cardiac investigation, $O R$ odds ratio, $B M I$ body mass index, $C V$ cardiovascular, $H x$ history, CCF chronic heart failure, $M I$ myocardial infarction, $C A D$ coronary artery disease, PVD peripheral vascular disease, CVA cerebrovascular accident, VTE venous thromboembolism, CKD chronic kidney disease, COPD chronic obstructive pulmonary disease, OSA obstructive sleep apnoea, ECG electrocardiogram, Ix investigation

patients (30.7\%) during pre-operative cardiology review (Additional Table 1).

In the total population, six patients did not proceed with surgery following cardiology review: one had severe valvular disease identified on history, one had ECG evidence of a prior infarction not previously known, two had evidence of severe stenosis on CTCA, and three were cancelled due to non-cardiac reasons. Postoperative arrhythmia (atrial fibrillation) was reported in seven patients, with no other cardiac complications post-operatively.

\section{Additional cardiac investigation (ACl)}

One hundred thirty-eight patients underwent $\mathrm{ACI}$ following pre-operative cardiology review of ECG/ECHO. The mean age was $72.3 \pm 8.7$ years, and age was found to be significantly associated with ACI $(p<0.001)$ (Table 1$)$. Hypertension, previous cardiac history and $\mathrm{FHx}$ of cardiac 
disease were more prevalent in this cohort compared to the total patient population, and these variables were all significant for additional cardiac investigation $(p=0.001$, $p=0.001$ and $p<0.001$, respectively) (Table 1 ). More specifically, a history of CAD or CVA was significantly associated with ACI ( $p=0.004$ and $p<0.001$, respectively). An ECG abnormality, such as heart block, atrial fibrillation, evidence of prior ischaemic events, or ectopic beats, was detected in $38.4 \%$ patients that went on to have additional cardiac investigation $(p=0.010)$. The mean $\mathrm{EF}$ was similar to the mean for the total patient population $(62.0 \%$ vs. $63.8 \%)$, and EF was significantly associated with ACI ( $p=$ 0.005) (Table 1).

According to the logistic regression, age (OR 1.06, 95\% CI $1.03-1.09, p<0.001$ ), CVA (OR 3.83, 95\% CI $1.26-$ $11.61, p=0.018$ ) and FHx of CVD (OR 4.20, 95\% CI $2.27-7.80, p<0.001)$ significantly predicted the need for $\mathrm{ACI}$, while increased EF was predictive of cardiologist not referring for further investigation (OR 0.94, 95\% CI $0.91-0.97, p<0.001$ ) (Table 2). Hypertension and coronary artery disease increased the likelihood of requiring ACI 1.63-fold and 1.67-fold, respectively; however, these risk factors were not significant predictors $(p=0.080$ and $p=0.106$, respectively).

\section{Abnormality detected upon additional cardiac investigation (ADACI)}

Fifty patients were found to have an abnormality upon additional cardiac investigation. The mean age was 73.3 \pm 8.4 years, and age was found to be significantly associated with the incidence of an abnormal cardiac finding ( $p=0$.004) (Table 3). Male gender was more prevalent compared with the total cohort (70\% vs. $54 \%)$ and was

Table 2 Multivariate logistic regression for additional cardiac investigation

\begin{tabular}{|c|c|c|c|c|c|c|}
\hline \multirow[b]{2}{*}{ Predictors } & \multirow[b]{2}{*}{$\beta$} & \multirow[b]{2}{*}{ S.E } & \multirow[b]{2}{*}{$p$} & \multirow[b]{2}{*}{ OR } & \multicolumn{2}{|c|}{$95 \% \mathrm{Cl}$} \\
\hline & & & & & Low & High \\
\hline \multicolumn{7}{|l|}{ Demographics } \\
\hline Age & 0.06 & 0.02 & $<0.001$ & 1.06 & 1.03 & 1.09 \\
\hline HTN & 0.49 & 0.28 & 0.080 & 1.63 & 0.94 & 2.83 \\
\hline \multicolumn{7}{|l|}{ Cardiac history } \\
\hline CAD & 0.51 & 0.32 & 0.106 & 1.67 & 0.90 & 3.12 \\
\hline CVA & 1.34 & 0.57 & 0.018 & 3.83 & 1.26 & 11.61 \\
\hline FHx of CVD & 1.44 & 0.32 & $<0.001$ & 4.20 & 2.27 & 7.80 \\
\hline \multicolumn{7}{|l|}{ Baseline cardiac IX } \\
\hline ECG abnormality & 0.29 & 0.28 & 0.309 & 1.34 & 0.77 & 2.33 \\
\hline Ejection fraction & -0.06 & 0.02 & $<0.001$ & 0.94 & 0.91 & 0.97 \\
\hline
\end{tabular}

Multivariate logistic regression results with adjusted odds ratios (OR) and associated $95 \%$ confidence intervals $(\mathrm{Cl})$. Significant predictor variables are indicated by $p<0.05$

HTN hypertension, CAD coronary artery disease, CVA cerebrovascular accident, $F H x$ family history, CVD cardiovascular disease, $I x$

investigation, ECG electrocardiogram significantly associated with ADACI $(p=0.015)$, as were current and ex-smokers (both $p=0.009$ ) (Table 3). In contrast to the ACI group, cardiac history was not significantly associated with detection of an abnormality on additional cardiac investigation $(p=0.601)$. However, CVA and FHx of CVD were significant $(p=0.010$ and $p$ $=0.014$, respectively). ECG abnormality $(p=0.005)$ and $\mathrm{EF}(p<0.001)$ from baseline cardiac assessment were also both significantly associated with ADACI (Table 3).

A total of 17 patients in the ADACI group had a surgical delay by the cardiologist to undergo pre-operative cardiac optimisation, whereas the remaining patients were able to be optimised without surgical delay. In the delayed group, 12 had an ECG abnormality, either atrial fibrillation or heart block, and five had an $\mathrm{EF}<50 \%$ at baseline. Consequently, 15 had an abnormal angiogram and two had an abnormal MIBI.

Logistic regression revealed increased age, male gender, FHx of CVD, and reduced EF significantly predicted detection of a cardiac abnormality pre-operatively (Table 4). For every increased year in age, there was a significant $7 \%$ increase in ADACI (OR 1.07; 1.02-1.12 95\% Cl, $p=$ 0.003), while every percentage increase in EF reduced the likelihood of finding an abnormality by $7 \%$ (OR 0.93 ; $0.89-0.9795 \% \mathrm{Cl}, p<0.001)$. Male gender was also a significant predictor for ADACI (OR 2.22; 1.03-4.79 95\% Cl, $p=0.042)$. FHx of CVD was a strong predictor and significantly increased the likelihood of finding a cardiac abnormality 3.6-fold (OR 3.61; 1.65-7.89 95\% CI, $p=0.001$ ). Smoking status and history of CVA did not demonstrate any significant predictive capacity (Table 4).

To further validate these findings, significant predictors for ADACI were evaluated in a predictive model using ROC curve analysis. EF and Age were normalised against the mean of the cohort, with a Mean EF of $63.8 \%$ and a Mean Age of 69.9 years. As outlined in the "Methods" section, the model weighted the influence of the predictor variables based on the odds ratio produced by the logistic regression, as follows: (2* Gender) $+\left(3^{*}\right.$ FHx of CVD) + (Mean EF/Patient EF) + (Age/Mean Age). This model produced a cut-off score of 3.99 and maintained reasonable predictive capacity $(\mathrm{c}$-statistic $=$ 0.71 ), with $70 \%$ sensitivity and $65 \%$ specificity, and good calibration $(p>0.05)$.

\section{Discussion}

Cardiovascular complication continues to represent up to $20 \%$ of all major post-operative complication following TJA $[12,14]$ and will become increasingly important with an ageing, increasingly obese, and more medically complex arthroplasty population $[12,17,18]$. Pre-operative cardiology optimisation is essential to reduce post-operative cardiac events. However, no validated, orthopaedic-specific cardiac risk stratification tools are available to guide 
Table 3 Risk factors associated with abnormality detected upon additional cardiac investigation

\begin{tabular}{|c|c|c|c|c|c|c|c|c|}
\hline & \multicolumn{2}{|l|}{$\mathrm{ADACl}$} & \multicolumn{6}{|c|}{ Statistics } \\
\hline & Yes $(n=50)$ & No $(n=324)$ & $x^{2}$ & $p$ value & z score & Crude OR & $95 \% \mathrm{Cl}$ & $p$ value \\
\hline \multicolumn{9}{|l|}{ Demographics } \\
\hline Age (years) & $73.3 \pm 8.4$ & $69.4 \pm 9.0$ & - & 0.004 & - & 1.05 & $1.02-1.09$ & 0.005 \\
\hline Male & 35 (70\%) & $167(51.5 \%)$ & 5.94 & 0.015 & 2.4 & 2.19 & $1.15-4.17$ & 0.020 \\
\hline Female & 15 (30\%) & $157(48.5 \%)$ & 5.94 & 0.015 & -2.4 & 0.46 & $0.24-0.87$ & 0.020 \\
\hline BMl & $31.4 \pm 5.9$ & $31.2 \pm 5.3$ & - & 0.880 & - & 1.01 & $0.95-1.06$ & 0.870 \\
\hline \multicolumn{9}{|l|}{ Past medical Hx } \\
\hline Diabetes & $9(18 \%)$ & $47(14.5 \%)$ & 0.42 & 0.519 & 0.6 & 1.30 & $0.59-2.84$ & 0.520 \\
\hline Hypertension & 37 (74\%) & 207 (63.9\%) & 1.95 & 0.162 & 1.4 & 1.61 & $0.82-3.15$ & 0.170 \\
\hline Hypercholesterolaemia & $30(60.0 \%)$ & $186(57.4 \%)$ & 0.12 & 0.730 & 0.3 & 1.11 & $0.61-2.04$ & 0.730 \\
\hline Smoking & $6(12.0 \%)$ & $16(4.9 \%)$ & 9.52 & 0.009 & 2.0 & 3.39 & $1.22-9.42$ & 0.020 \\
\hline Ex-smoker & $18(36.0 \%)$ & $73(22.5 \%)$ & 9.52 & 0.009 & 2.1 & 2.23 & $1.16-4.29$ & 0.020 \\
\hline Alcohol & $6(12.0 \%)$ & $36(11.1 \%)$ & 0.03 & 0.853 & 0.2 & 1.09 & $0.43-2.74$ & 0.850 \\
\hline Asthma & 7 (14.0\%) & $37(11.4 \%)$ & 0.28 & 0.598 & 0.5 & 1.26 & $0.53-3.01$ & 0.600 \\
\hline COPD & $2(4.0 \%)$ & $12(3.7 \%)$ & 0.01 & 0.918 & 0.1 & 1.08 & $0.24-4.99$ & 0.920 \\
\hline OSA & $4(8.0 \%)$ & 39 (12.0\%) & 0.69 & 0.405 & -0.8 & 0.64 & $0.22-1.86$ & 0.410 \\
\hline \multicolumn{9}{|l|}{ Past CV Hx } \\
\hline Cardiac Hx total & 16 (32\%) & $116(35.8 \%)$ & 0.27 & 0.601 & -0.5 & 0.84 & $0.45-1.59$ & 0.600 \\
\hline CCF & $0(0.0 \%)$ & $8(2.5 \%)$ & 1.26 & 0.261 & -1.1 & 0.37 & $0.02-6.49$ & 0.500 \\
\hline $\mathrm{Ml}$ & $6(12.0 \%)$ & $22(6.8 \%)$ & 1.70 & 0.193 & 1.3 & 1.87 & $0.72-4.87$ & 0.200 \\
\hline$C A D$ & $11(22 \%)$ & $63(19.4 \%)$ & 0.18 & 0.673 & 0.4 & 1.14 & $0.56-2.34$ & 0.710 \\
\hline Valvular disease & $5(10.0 \%)$ & $20(6.2 \%)$ & 1.02 & 0.313 & 1.0 & 1.69 & $0.60-4.73$ & 0.320 \\
\hline Arrhythmia & $6(12.0 \%)$ & 49 (15.1\%) & 0.34 & 0.562 & -0.6 & 0.79 & $0.32-1.94$ & 0.610 \\
\hline PVD & $2(4.0 \%)$ & $5(1.5 \%)$ & 1.42 & 0.233 & 1.2 & 2.66 & $0.50-14.09$ & 0.250 \\
\hline CVA & $8(16.0 \%)$ & 19 (5.9\%) & 6.64 & 0.010 & 2.6 & 2.45 & $1.04-5.74$ & 0.040 \\
\hline VTE & $4(8.0 \%)$ & $10(3.1 \%)$ & 2.90 & 0.088 & 1.7 & 2.73 & $0.82-9.07$ & 0.100 \\
\hline CKD & $3(6.0 \%)$ & $20(6.2 \%)$ & 0.47 & 0.790 & 0.0 & 0.97 & $0.28-3.37$ & 0.970 \\
\hline Family $\mathrm{Hx}$ of cardiac disease & $16(32.0 \%)$ & $56(17.3 \%)$ & 6.03 & 0.014 & 2.5 & 2.25 & $1.16-4.36$ & 0.020 \\
\hline \multicolumn{9}{|l|}{ Baseline cardiac Ix } \\
\hline ECG abnormality & $24(48.0 \%)$ & $91(28.1 \%)$ & 8.07 & 0.005 & 2.8 & 2.36 & $1.29-4.33$ & 0.005 \\
\hline Ejection fraction (\%) & $58.9 \pm 11.6$ & $64.6 \pm 7.3$ & - & 0.002 & - & 0.93 & $0.89-0.96$ & $<0.001$ \\
\hline
\end{tabular}

Numerical predictor variables (age, BMI, ejection fraction) are presented as mean \pm standard deviation and were assessed using an independent $t$ test. Categorical predictor variables are presented as frequencies and were assessed using chi-squared test of independence. The crude ratio and associated $95 \% \mathrm{Cl}$ are also reported. Significant predictor variables are indicated by $p<0.05$

$A D A C l$ abnormality detected upon additional cardiac investigation, $O R$ odds ratio, $B M I$ body mass index, $C V$ cardiovascular, $H x$ history, $C C F$ chronic heart failure, $M I$ myocardial infarction, CAD coronary artery disease, $P V D$ peripheral vascular disease, CVA cerebrovascular accident, VTE venous thromboembolism, CKD chronic kidney disease, COPD chronic obstructive pulmonary disease, OSA obstructive sleep apnoea, Ix investigation, ECG electrocardiogram

orthopaedic referral of patients to cardiology [3, 19-21]. There has been no previous research on cardiac referral for the asymptomatic preoperative TJA population in which cardiac disease not yet exhibiting clinical manifestation but capable of contributing to risk of postoperative cardiac complication may exist. Over-referral is unsustainable $[4,22-24]$ and may unnecessarily delay surgery $[25,26]$; however under-referral may lead to missed pre-operative optimisation for asymptomatic patients who otherwise would have benefited, and may contribute to post-operative cardiac complication.

The need for a pre-operative risk stratification tool for lower limb arthroplasty was recognised by Waterman et al., who proposed the TJA Cardiac Risk Index Score [3]. Though the results of this study are promising, their use of the National Surgical Quality Improvement Program (NSQIP) database limited their analysis as only risk factors recorded in the database could be evaluated, and 
Table 4 Multivariate logistic regression for abnormality detected upon additional cardiac investigation

\begin{tabular}{llllllll}
\hline & & & & & \multicolumn{2}{c}{$\mathbf{9 5 \%} \mathbf{C l}$} \\
\cline { 7 - 8 } Predictors & $\boldsymbol{\beta}$ & S.E & $\mathbf{p}$ & OR & Low & High \\
\hline Demographics & & & & & & \\
$\quad$ Age & 0.07 & 0.02 & 0.003 & 1.07 & 1.02 & 1.12 \\
$\quad$ Gender & 0.80 & 0.39 & 0.042 & 2.22 & 1.03 & 4.79 \\
$\quad$ Current smoker & 0.80 & 0.67 & 0.231 & 2.22 & 0.60 & 8.17 \\
$\quad$ Ex-smoker & -0.46 & 0.40 & 0.250 & 0.63 & 0.29 & 1.38 \\
Cardiac history & & & & & & \\
$\quad$ CVA & 1.01 & 0.59 & 0.087 & 2.74 & 0.86 & 8.71 \\
Family history & & & & & & \\
$\quad$ FHx of CVD & 1.28 & 0.40 & 0.001 & 3.61 & 1.65 & 7.89 \\
Baseline cardiac Ix & & & & & & \\
$\quad$ ECG abnormality & 0.23 & 0.39 & 0.556 & 1.26 & 0.59 & 2.68 \\
$\quad$ Ejection fraction & -0.08 & 0.02 & $<0.001$ & 0.93 & 0.89 & 0.97 \\
\hline
\end{tabular}

Multivariate logistic regression results for abnormality detected upon additional cardiac investigation with adjusted odds ratios (OR) and associated $95 \%$ confidence intervals (CI). Significant predictor variables are indicated by $p$ $<0.05$

CVA cerebrovascular accident, $F H x$ family history, CVD cardiovascular disease, Ix investigation, ECG electrocardiogram

patient follow-up was limited to only 30 days postoperative $[3,27]$. Improved cohort design and broader evaluation of risk factors is required to determine all risk factors that should guide pre-operative cardiology referral. Risk factors identified by the recent review by Elsiwy et al. were evaluated in this series and tested for their capacity to predict the need for additional cardiac investigation pre-operatively and abnormality detected upon additional cardiac investigation. Uniquely in this study, all patients, including asymptomatic patients without known cardiac disease, were referred to cardiology preoperatively for ECG/ECHO, thereby avoiding the bias of selective referral of only higher-risk patients. Furthermore, in contrast to all previous literature, risk factors in this study were not measured against their capacity to predict post-operative cardiac events, but rather the ability to predict cardiologist referral for additional investigation pre-operatively, and cardiac abnormalities detected during additional investigation, which may prompt cardiac optimisation and/or surgical delay. The additional advantage of examining these outcome variables in a population that has all been referred to cardiology is that the overall cardiovascular profile of an asymptomatic population can be determined, patients with silent disease can be identified and risk factors which can better guide pre-operative cardiac referral can be determined.

Importantly, our cohort had a demographic profile comparable to previous arthroplasty populations described in the literature. The mean age and the cardiac complication rate $(1.87 \%)$ was similar to the Waterman study $[3,14]$, while the cardiovascular risk profile of our cohort was similar to that described by Łęgosz et al. in their analysis of cardiovascular risk of arthroplasty populations [4]. No patients in our series suffered a major cardiac event or post-operative mortality, reflecting the experience and expertise of the orthopaedic surgeon. Given the small cohort size of 374 patients, this is also consistent with the low rates of mortality $(0.18 \%)$ and incidence of major cardiac complication (myocardial infarction or cardiac arrest) previously reported $(0.2 \%)[2,3]$, but may also be due to the fact that all patients were referred for cardiology review and optimisation pre-operatively. The importance of this is further demonstrated by the finding of ischaemic lesions during $\mathrm{ACI}$ in 17 'asymptomatic' patients that went on to have surgical delays.

By assessing predictors of additional cardiac investigation, this study highlights for the first time the risk factors that cardiologists are more likely to utilise for pre-operative risk stratification of TKA and THA patients. In this series, cardiologists were more likely to conduct additional cardiac investigation based on increasing age, FHx of CVD, previous CVA, and reduced EF. Of interest, although CVA was considered important by the cardiologists, it did not show a significant predictive capacity for detecting cardiac abnormality. The link between CVA and post-operative cardiac complication is controversial [14]; however, these results support previous literature in which CVA was not significantly associated with post-operative cardiac complication $[2,7]$. In contrast, reduced EF was a consistent predictor for both $\mathrm{ACI}$ and $\mathrm{ADACI}$, though current literature does not support the use of routine echocardiogram in preoperative cardiovascular screening [28, 29].

For cardiac risk stratification, the detection of a cardiac abnormality is most relevant, and these patients are most likely to benefit from pre-operative cardiac optimisation since they have demonstrated cardiac disease. Similar to multiple studies showing a strong association between increasing age and post-THA and TKA cardiac complication [2, 3, 7, 10-12], our analysis demonstrated a significant predictive capacity of every year of increase in age for the detection of a cardiac abnormality preoperatively. Male gender was also a significant predictor for ADACI and should therefore be considered in preoperative risk stratification. Interestingly, this was not reported by Waterman et al., although the influence of male gender on increasing the risk of cardiovascular complication is well established in the literature $[10,13]$. A 3.6-fold increased risk of detecting a cardiac abnormality with a FHx of CVD suggests that this risk factor should also be considered in pre-operative cardiac risk stratification. Notably, this risk factor has not been 
considered in this context in previous literature and was not addressed in the review by Elsiwy et al. [14]. Although family history is a long-established risk factor for cardiovascular disease, perhaps it has not been considered in previous arthroplasty studies due to its nonmodifiable nature [30]. In contrast to having a family history, patient cardiac history was not a significant predictor for ADACI. While an association between previous cardiac history and post-operative cardiac complication has been reported [2, 3, 7, 11, 12, 31], it is possible that patients in the current study were previously optimised so the influence of their cardiac history had been mitigated or reversed. This is also potentially true of patients with diabetes, hypertension, hypercholesterolaemia and other risk factors linked to cardiac complication post-TJA in the literature that did not show significance in our series.

These findings that increased age, male gender, FHx of CVD and decreased ejection fraction predicted cardiac abnormality were internally validated in a predictive model that produced a statistically significant c-statistic $>0.70$ which was equivalent to the model proposed by Waterman [3], and had reasonable discriminative capacity and no evidence of statistically significant lack of fit $(p>0.05)$ [32]. The proposed model demonstrates an innovative method of producing an algorithm that can be used to risk-stratify TJA patients and guide preoperative cardiac referral. In contrast to the methods previously described by Waterman et al. [3] and several other surgical risk calculators $[19,21,33]$ where various iterations are tested until the highest c-statistic is obtained, risk factors included in the our model were weighted based on their odds ratios, taking into consideration the individual influence of each risk factor.

\section{Limitations}

The authors acknowledge several limitations that prevent translation of the proposed risk stratification model into clinical practice at this time. Due to the small population, additional risk factors that occur in low incidence within this population were unable to be accurately investigated, and continuous variables age and EF were not able to be stratified to evaluate specific ranges. Small sample size may also explain why being a previous or current smoker showed significant association but did not achieve significant predictive capacity in the regression model, in contrast to current literature [14]. Furthermore, some parameters such as the American Society of Anesthesiologists (ASA) score, which has previously shown significance in the literature, were not available in this data set [3]. However, this study avoids the inherent limitations of larger database-driven studies already discussed and provides a unique cohort of asymptomatic patients, who were all referred to cardiology prior to TKA or THA. Another limitation is the inclusion of $\mathrm{EF}$ as a predictive risk factor. Since routine echocardiography is not currently evidence-based practice [29], not all patients will have this measurement readily available for pre-operative risk stratification. There is evidence, however, that echocardiography has a role in pre-operative evaluation of higher risk patients [29]. Given the results of the current study which suggest that even asymptomatic TJA patients may be high risk if they are over 70 , male and have a family history of cardiovascular disease, the benefit of echocardiography in this subset of patients needs further evaluation. While the bias of cardiac referral has been removed in this study, there is still potential bias in the provision of additional cardiac investigation by the cardiologist. We attempted to minimise this bias by including seven different cardiologists, and the consistency between regression models of $\mathrm{ACI}$ and ADACI suggest appropriate selection of patients for additional cardiological intervention in this study. Finally, the categorical representation of risk factors such as hypertension, diabetes and hypercholesterolaemia does not distinguish between patients in whom these comorbidities are well controlled versus poorly controlled. Future studies should aim to collect objective parameters such as systolic blood pressure, blood sugar level and total cholesterol so as to improve assessment of the impact of these comorbidities.

\section{Clinical implications and future research}

Despite these limitations, the predictive model presented here has broader implications for clinical practice which may be used to assist orthopaedic surgeons with more evidence-based referral to cardiology prior to TJA. The finding that a family history of cardiovascular disease had the greatest power in our model suggests the presence of this risk factor should prompt the surgeon to refer for pre-operative cardiac assessment. In the absence of FHx of CVD, male patients should also be referred given that a male of mean age and with a mean EF would reach the model cut-off score. This study also highlights the value of age and ejection fraction in determining which patients would benefit from pre-operative cardiology referral prior to undergoing TKA or THA. Additionally, the proposed model may serve as an example for future studies attempting to create a TJA-specific pre-operative cardiac referral tool, particularly for asymptomatic patients. Future investigations in this area should employ larger and more tailored patient cohorts that are specifically designed to develop a comprehensive tool that considers all risk factors associated with cardiac complication post-TJA that have been identified in the literature. 


\section{Conclusions}

As arthroplasty procedures increase exponentially, a validated pre-operative cardiac risk stratification tool is crucial to assist decision-making by orthopaedic surgeons with regard to patient referral for pre-operative cardiac optimisation. Increasing age, male gender, FHx of CVD, and reduced EF were identified as significant predictors for detection of cardiac abnormality in asymptomatic patients on pre-operative work-up. Future predictive models require larger cohorts which evaluate all potential risk factors of post-operative cardiac complication. Targeted pre-operative cardiac optimisation will not only reduce the risk of post-operative cardiac complication, but also has the potential to reduce health expenditure and surgical delays.

\section{Supplementary Information}

The online version contains supplementary material available at https://doi. org/10.1186/s13018-020-02042-5.

Additional file 1. Table 1. Demographic characteristics and risk factors of patients in the total patient population. Supplementary Figure 1: Inclusion and exclusion criteria. TKA = total knee arthroplasty; THA = total hip arthroplasty; ECG = electrocardiogram; ECHO = echocardiogram. Supplementary Figure 2: A simplified flowchart of the analytical process. All data analysis used SPSS, V.24, P $<0.05$ statistically significant. TKA = Total Knee Arthroplasty; THA = Total Hip Arthroplasty.

\section{Abbreviations}

ACl: Additional cardiac investigation; ADACl: Abnormality detected on additional cardiac investigation; ASA: American Society of Anesthesiologists; BMI: Body mass index; CAD: Coronary artery disease; Cl: Confidence interval; CTCA: CT-coronary angiography; CVA: Cerebrovascular accident; CVD: Cardiovascular disease; ECG: Electrocardiogram; ECHO: Echocardiogram; EF: Ejection fraction; FHx: Family history; HTN: Hypertension; MIBI: Myocardial perfusion scan; NSQIP: National Surgical Quality Improvement Program; OR: Odds ratio; ROC: Receiver operator characteristics; THA: Total hip arthroplasty; TJA: Total joint arthroplasty; TKA: Total knee arthroplasty

\section{Acknowledgements}

The authors would like to acknowledge the valuable contributions of Mrs Andrea Grant for administrative support, Mrs. Alicia Harris for assistance with data collection, Dr. Jodie Morris for editorial assistance, and the Orthopaedic Research Institute of Queensland (ORIQL) and College of Medicine and Dentistry, James Cook University, for the ongoing research support.

\section{Authors' contributions}

YE, KH and HL developed the research question and study design. YE and TS completed the data collection. YE, KD and $\mathrm{HL}$ completed the data analysis and interpretation. YE drafted the manuscript, which was edited and reviewed by $\mathrm{KD}, \mathrm{KH}, \mathrm{MW}$ and $\mathrm{HL}$. The authors have read and approved the final manuscript.

\section{Funding}

This research did not receive any specific grant from funding agencies in the public, commercial or not-for-profit sectors.

\section{Availability of data and materials}

The datasets used and analysed during the current study are available from the corresponding author on reasonable request.

\section{Ethics approval and consent to participate}

This study was approved by the Mater Health Services North Queensland Human Research Ethics Committee (Approval: MHS20180424-01). A waiver of consent was approved in accordance with the National Statement 2.3.10 that this constitutes a negligible or low risk to participants who have already received standard of care.

Consent for publication

Not applicable

\section{Competing interests}

The authors declare that they have no competing interests.

\section{Author details}

${ }^{1}$ College of Medicine and Dentistry, James Cook University, 1 James Cook Drive, Townsville, QLD 4811, Australia. ${ }^{2}$ Orthopaedic Research Institute of Queensland, 7 Turner Street, Townsville, QLD 4812, Australia. ${ }^{3}$ College of Healthcare Sciences, James Cook University, 1 James Cook Drive, Townsville, QLD 4811, Australia. ${ }^{4}$ School of Medicine, University of Tasmania, Medical Science Precinct, 17 Liverpool Street, Hobart, TAS 7000, Australia.

Received: 3 September 2020 Accepted: 28 October 2020

Published online: 10 November 2020

\section{References}

1. Memtsoudis SG. González Della Valle A, Besculides MC, Gaber L, Sculco TP. In-hospital complications and mortality of unilateral, bilateral, and revision TKA: based on an estimate of 4,159,661 discharges. Clinical orthopaedics and related research. 2008;466(11):2617-27.

2. Shah CK, Keswani A, Boodaie BD, Yao DH, Koenig KM, Moucha CS. Myocardial infarction risk in arthroplasty vs arthroscopy: how much does procedure type matter? Journal of Arthroplasty. 2017;32(1):246-51.

3. Waterman BR, Belmont PJ Jr, Bader JO, Schoenfeld AJ. The Total Joint Arthroplasty Cardiac Risk Index for predicting perioperative myocardial infarction and cardiac arrest after primary total knee and hip arthroplasty. Journal of Arthroplasty. 2016:31(6):1170-4.

4. Łegosz P, Kotkowski M, Płatek AE, Małdyk P, Krzowski B, Rys A, et al. Assessment of cardiovascular risk in patients undergoing total joint alloplasty: The CRASH-JOINT study. Kardiologia Polska. 2017;75(3):213-20.

5. Basilico FC, Sweeney G, Losina E, Gaydos J, Skoniecki D, Wright EA, et al. Risk factors for cardiovascular complications following total joint replacement surgery. Arthritis and Rheumatism. 2008;58(7):1915-20.

6. Avram V, Petruccelli D, Winemaker M, de Beer J. Total joint arthroplasty readmission rates and reasons for 30-day hospital readmission. Journal of Arthroplasty. 2014;29(3):465-8.

7. Thornqvist C, Gislason GH, Køber L, Jensen PF, Torp-Pedersen C, Andersson C. Body mass index and risk of perioperative cardiovascular adverse events and mortality in 34,744 Danish patients undergoing hip or knee replacement. Acta Orthopaedica. 2014;85(5):456-62.

8. Tabatabaee RM, Rasouli MR, Rezapoor M, Maltenfort MG, Ong AC, Parvizi J. Coronary revascularization and adverse events in joint arthroplasty. Journal of Surgical Research. 2015;198(1):135-42.

9. Lu N, Misra D, Neogi T, Choi HK, Zhang Y. Total joint arthroplasty and the risk of myocardial infarction: a general population, propensity scorematched cohort study. Arthritis \& rheumatology (Hoboken, NJ). 2015; 67(10):2771-9.

10. Menendez ME, Memtsoudis SG, Opperer M, Boettner F. Gonzalez Della Valle A. A nationwide analysis of risk factors for in-hospital myocardial infarction after total joint arthroplasty. International Orthopaedics. 2015;39(4):777-86.

11. Feng B, Lin J, Jin J, Qian W, Cao S, Weng X. The effect of previous coronary artery revascularization on the adverse cardiac events ninety days after total joint arthroplasty. Journal of Arthroplasty. 2018;33(1):235-40.

12. Belmont PJ, Goodman GP, Kusnezov NA, Magee C, Bader JO, Waterman BR, et al. Postoperative myocardial infarction and cardiac arrest following primary total knee and hip arthroplasty: rates, risk factors, and time of occurrence. Journal of Bone and Joint Surgery - American Volume. 2014; 96(24):2025-31.

13. Robinson J, Shin JI, Dowdell JE, Moucha CS, Chen DD. Impact of gender on 30-day complications after primary total joint arthroplasty. Journal of Arthroplasty. 2017;32(8):2370-4

14. Elsiwy Y, Jovanovic I, Doma K, Hazratwala K, Letson $\mathrm{H}$. Risk factors associated with cardiac complication after total joint arthroplasty of the hip and knee: a systematic review. Journal of Orthopaedic Surgery and Research. 2019;14(1):15. 
15. Fleisher LA, Fleischmann KE, Auerbach AD, Barnason SA, Beckman JA, Bozkurt B, et al. 2014 ACC/AHA guideline on perioperative cardiovascular evaluation and management of patients undergoing noncardiac surgery: executive summary: a report of the American College of Cardiology/American Heart Association Task Force on Practice Guidelines. Circulation. 2014;130(24):2215-45.

16. O'brien RM. A caution regarding rules of thumb for variance inflation factors. Quality \& Quantity. 2007;41(5):673-90.

17. Belmont PJ Jr, Goodman GP, Hamilton W. aterman BR, Bader JO, Schoenfeld AJ. Morbidity and mortality in the thirty-day period following total hip arthroplasty: risk factors and incidence. Journal of Arthroplasty. 2014;29(10):2025-30.

18. Jorgensen CC, Petersen MA, Kehlet $\mathrm{H}$. Preoperative prediction of potentially preventable morbidity after fast-track hip and knee arthroplasty: a detailed descriptive cohort study. BMJ Open. 2016;6(1).

19. Peterson B, Ghahramani M, Harris S, Suchniak-Mussari K, Bedi G, Bulathsinghala $C$, et al. Usefulness of the myocardial infarction and cardiac arrest calculator as a discriminator of adverse cardiac events after elective hip and knee surgery. American Journal of Cardiology. 2016;117(12):1992-5.

20. Gaston M, Amin A, Clayton R, Brenkel I. Does a history of cardiac disease or hypertension increase mortality following primary elective total hip arthroplasty? The Surgeon. 2007:5(5):260-5.

21. Marya S, Amit P, Singh C. Impact of Charlson indices and comorbid conditions on complication risk in bilateral simultaneous total knee arthroplasty. The Knee. 2016;23(6):955-9.

22. Sheffield KM, MCAdams PS, Benarroch-Gampel J, Goodwin JS, Boyd CA, Zhang $D$, et al. Overuse of preoperative cardiac stress testing in medicare patients undergoing elective noncardiac surgery. Annals of Surgery. 2013; 257(1):73-80.

23. Chou R, Arora B, Dana T, Fu R, Walker M, Humphrey L. Screening asymptomatic adults with resting or exercise electrocardiography: a review of the evidence for the U.S. preventive services task force. Annals of Internal Medicine. 2011;155(6).

24. Wijeysundera DN, Beattie WS, Elliot RF, Austin PC, Hux JE, Laupacis A. Non-invasive cardiac stress testing before elective major non-cardiac surgery: population based cohort study. BMJ (Online). 2010;340(7740): 252.

25. Groot M, Spronk A, Hoeks S, Stolker R, van Lier F. The preoperative cardiology consultation: indications and risk modification. Netherlands Heart Journal. 2017;25(11):629-33.

26. Coffman J, Tran T, Quast T, Berlowitz MS, Chae SH. Cost conscious care: preoperative evaluation by a cardiologist prior to low-risk procedures. BMJ Open Quality. 2019;8(2):e000481.

27. Alluri RK, Leland $H$, Heckmann N. Surgical research using national databases. Annals of Translational Medicine. 2016;4(20).

28. Wijeysundera DN, Beattie WS, Karkouti K, Neuman MD, Austin PC, Laupacis A. Association of echocardiography before major elective non-cardiac surgery with postoperative survival and length of hospital stay: population based cohort study. Bmj. 2011;342:d3695.

29. Shim CY. Preoperative cardiac evaluation with transthoracic echocardiography before non-cardiac surgery. Korean journal of anesthesiology. 2017;70(4):390-7.

30. Friedlander $Y$, Siscovick DS, Weinmann S, Austin MA, Psaty BM, Lemaitre RN, et al. Family history as a risk factor for primary cardiac arrest. Circulation. 1998;97(2):155-60.

31. Curtis GL, Newman JM, George J, Klika AK, Barsoum WK, Higuera CA Perioperative outcomes and complications in patients with heart failure following total knee arthroplasty. Journal of Arthroplasty. 2018; 33(1):36-40.

32. Vanagas $G$. Receiver operating characteristic curves and comparison of cardiac surgery risk stratification systems. Interactive cardiovascular and thoracic surgery. 2004;3(2):319-22.

33. Fleisher LA, Beckman JA, Brown KA, Calkins H, Chaikof E, Fleischmann KE, et al. ACC/AHA 2007 guidelines on perioperative cardiovascular evaluation and care for noncardiac surgery: executive summary: a report of the American College of Cardiology/American Heart Association Task Force on practice guidelines (writing committee to revise the 2002 guidelines on perioperative cardiovascular evaluation for noncardiac surgery) developed in collaboration with the American Society of Echocardiography, American Society of Nuclear Cardiology,
Heart Rhythm Society, Society Of Cardiovascular Anesthesiologists, Society for Cardiovascular Angiography and Interventions, Society for Vascular Medicine and Biology, and Society for Vascular Surgery. Journal of the American College of Cardiology. 2007;50(17):1707-32.

\section{Publisher's Note}

Springer Nature remains neutral with regard to jurisdictional claims in published maps and institutional affiliations.
Ready to submit your research? Choose BMC and benefit from:

- fast, convenient online submission

- thorough peer review by experienced researchers in your field

- rapid publication on acceptance

- support for research data, including large and complex data types

- gold Open Access which fosters wider collaboration and increased citations

- maximum visibility for your research: over $100 \mathrm{M}$ website views per year

At $\mathrm{BMC}$, research is always in progress.

Learn more biomedcentral.com/submissions 